\title{
Exploration de la variabilité génétique présente dans cinq peuplements naturels de pin Laricio de Corse (Pinus nigra ssp. laricio
} var. corsicana Loud.)

\section{Portefaix}

INRA - Station d'amélioration des arbres forestiers, Ardon, 45160 Olivet, France

(reçu le 20 avril 1988; accepté le 18 octobre 1988)

Résumé - La variabilité génétique présente dans cinq peuplements naturels de pin laricio de Corse a été étudiée pour quelques prédicteurs de la croissance en volume et de la forme, à partir d'un test de descendances maternelles âgé de 11 ans, situé en forêt d'Orléans.

L'analyse factorielle discriminante met en évidence une variation clinale des caractères de croissance suivant un gradient altitudinal. Les peuplements représentant la tranche $700-1000 \mathrm{~m}$ se révèlent plus vigoureux que ceux situés entre 950 et $1200 \mathrm{~m}$ d'altitude.

La comparaison des cinq estimations de variances-covariances interfamilles, réalisée à l'aide du test de Kullback, conduit à rejeter l'hypothèse d'homogénéité des structures génétiques intrapeuplement.

La forte différenciation observée entre ces cinq populations naturelles voisines est discutée en faisant référence aux rôles potentiels des pressions de sélection naturelle et de la dérive génétique.

Pinus nigra ssp. laricio var. corsicana - variabilité génétique - héritabilité - corrélation génétique-population naturelle

Summary - Exploration of genetic variability of five natural stands of Corsican pine (Pinus nigra ssp. laricio var. corsicana Loud.). Studies on genetic variability existing in natural populations of Corsican pine are of major concern in developing an optimum tree breeding program.

A total of 97 wind-pollinated families grouped into 5 natural stands from the northern part of the Corsican island (Fig. 1, Table 1), were raised in a progeny test in Orleans forest and assessed for growth characteristics from age 2 till age 11 after plantation. Measurements of forking defects were also made for the years 1983, 1984 and 1985.

Two models of analysis of variance were used : the first one to compare stand means, the second one to compute for each stand estimates of genetic parameters (between-family variances, individual heritabilities, genetic correlations).

Phenotypical differences among population means, indicated by discriminant functions, were mainly explained by juvenile growth performances (Table II, Fig. 2, Table III). For these traits, a pattern of clinal variation might exist, depending on an elevational gradient.

Genetic parameters varied widely from one stand to another (Table IV, Table V). For growth characteristics, a negative relationship appeared between performance mean and between-family variance value. However, because of a lack of precision in variance components estimation, the 
hypothesis of homogeneity based on between-family variances, checked by Kullback test could not be rejected for a single given trait (Table VI).

Kullback test values computed with special groups of traits showed large discrepancies of genetic structure among the 5 natural stands (Table VII, Fig. 3). tions :

Results were discussed refering to two main causes of genetic differentiation of isolated popula-

- natural selection pressures, essentially rainfall conditions for Corsican pine in its native range;

- genetic drift and factors liable to reduce mean consanguinity rate as could probably be, fire, colonisation of neighbouring areas and human activity.

Pinus nigra ssp laricio var corsicana - natural population - genetic variability - genetic structure - heritability - genetic correlation

\section{Introduction}

L'exploration de la variabilité génétique présente dans différentes populations géographiques d'une même espèce forestière se révèle être un des outils les plus précieux à l'améliorateur :

- elle l'aide à décrire, voire à expliquer, certaines différences génétiques, physiologiques et morphologiques observées entre origines (Tigerstedt, 1974; Yang et al., 1977).

- elle permet d'évaluer le degré de spécialisation des différentes populations sous l'effet de facteurs directifs (migration, sélection naturelle ou artificielle) ou dispersifs (dérive génétique, régime de reproduction dans la population) (Bergmann, 1974; Rehfeldt, 1982; El Kassaby et al., 1981). Une telle approche des lois de variations génétiques à l'échelle géographique participe au lourd travail d'évaluation et de conservation des ressources génétiques d'une espèce.

- conduite sur des dispositifs expérimentaux adaptés, elle fournit les informations nécessaires à l'optimisation du programme d'amélioration, tant au niveau du choix de la population de base qu'à celui de la stratégie de sélection à adopter à court, moyen ou long terme (Birot et Christophe, 1979).

Les études de variabilité réalisées jusqu'à présent sur de nombreuses espèces forestières ont mis en œuvre les trois voies complémentaires suivantes:

- l'analyse de distributions alléliques dans les différentes populations grâce à l'utilisation de marqueurs génétiques : électrophorèse de protéines (Rudin, 1976); chromatographie de composés terpéniques (Squillace, 1976; Baradat et al., 1976);

- la description de la variabilité intrapopulation grâce à différentes méthodes d'analyse de données : analyse en composantes principales ou analyse factorielle discriminante (Guyon, 1980);

- la décomposition de la variance phénotypique suivant les différents niveaux de variabilité étudiés, grâce aux modèles de la génétique quantitative (Birot et Christophe, 1983a et b).

Notre contribution à l'exploration de la variabilité génétique du pin laricio de Corse s'oriente vers cette troisième voie par l'estimation des paramètres génétiques de cinq massifs naturels de lî̀le de Beauté, pour des prédicteurs de la croissance en volume et de la forme. 
Tableau I. Massifs naturels corses et descendances maternelles étudiés.

\begin{tabular}{lccl}
\hline Massif étudié & $\begin{array}{l}\text { Pluviométrie } \\
\text { annuelle moyenne } \\
\text { (a) }\end{array}$ & $\begin{array}{l}\text { Altitude moyenne } \\
\text { d'échantillonnage } \\
\text { (b) }\end{array}$ & $\begin{array}{l}\text { Nb. descendances } \\
\text { étudiées } \\
\text { (c) }\end{array}$ \\
\hline $\begin{array}{c}\text { Tartagine-Melaja } \\
\text { (Tart) }\end{array}$ & $800-950 \mathrm{~mm}$ & $750-1000 \mathrm{~m}$ & 20 \\
$\begin{array}{c}\text { Valdo-Niello } \\
\text { (Vald) }\end{array}$ & $1550-1750 \mathrm{~mm}$ & $950-1150 \mathrm{~m}$ & 30 \\
$\begin{array}{c}\text { Aitone-Evisa } \\
\text { (Aito) }\end{array}$ & $1600-1800 \mathrm{~mm}$ & $1000-1200 \mathrm{~m}$ & 20 \\
$\begin{array}{c}\text { Vizzavona } \\
\text { (Vizz) }\end{array}$ & $1400-1500 \mathrm{~mm}$ & $950-1150 \mathrm{~m}$ & 12 \\
$\begin{array}{c}\text { Noceta } \\
\text { (Noce) }\end{array}$ & $1100-1300 \mathrm{~mm}$ & $820-1100 \mathrm{~m}$ & 15 \\
\hline
\end{tabular}

\section{Matériel et Méthodes}

\section{Matériel végétal}

L'expérience analysée est un test de descendances maternelles implanté en 1974 en forêt d'Orléans sur un sol siliceux profond. Les 97 familles étudiées ont été récoltées en 1972 sur les arbres échantillonnés au hasard dans cinq grands massifs naturels corses (Tableau I et Fig. 1):

- le massif de Tartagine-Melaja couvrant les versants nord des deux vallées de la Tartagine et de la Melaja. II s'agit là des peuplements naturels situés aux altitudes les plus basses (700-1 $400 \mathrm{~m}$ ) dans un faciès relativement sec;

- le massif de Valdo-Niello localisé sur le versant nord de la haute vallée du Golo entre 950 ef $1450 \mathrm{~m}$ d'altitude. Site forestier remarquable, il est à l'origine de la réputation du pin laricio de Corse;

- le massif d'Aitone-Evisa séparé du massif de Valdo-Niello par le col de Vergio. Les boisements principaux de pin laricio sont situés entre 900 ef $1300 \mathrm{~m}$ en exposition nord-ouest. La pluviométrie moyenne y est élevée, comme en témoigne la présence de hêtres et de sapins autochtones en limite supérieure des peuplements;

- le massif de Vizzavona situé au cceur de líle, au pied du Monte Renoso. II s'étend entre 725 et $1150 \mathrm{~m}$ de part et d'autre de l'Agnone. En versant ouest, le pin laricio de Corse est accompagné en sous-étage par un taillis de hêtre;
- le massif de Noceta, formé des quatre forêts continues et homogènes de Noceta, Rospigliani, Murracciole et Rospa-Sorba. Le pin laricio s'y développe à l'état pur entre 750 et $1100 \mathrm{~m}$ le long de pentes assez fortes.

Pour diminuer les risques d'apparentement, une distance minimale de $20 \mathrm{~m}$ était requise entre deux arbres-mères.

L'échantillonnage s'est limité aux parcelles les plus représentatives des séries de production de chaque peuplement. Le nombre moyen de descendances maternelles collectées par population constitue néanmoins un facteur limitant de cette étude. En effet, un échantillon de 30 familles est jugé minimal par plusieurs auteurs pour estimer avec précision les paramètres génétiques d'une population (Cotterrill et James, 1984).

\section{Dispositif expérimental et caractères mesurés}

A linstallation du dispositif, chaque descendance est représentée par 70 individus répartis dans des blocs complets par parcelle unitaire linéaire de 2 arbres.

Une notation individuelle de survie, réalisée après deux ans de plantation, a permis d'estimer le taux moyen de reprise (SURVIE.2) des différentes unités génétiques testées.

Au cours des onze premières années de plantation, la croissance en volume a été appréciée à l'aide de deux types de prédicteurs juvéniles : 


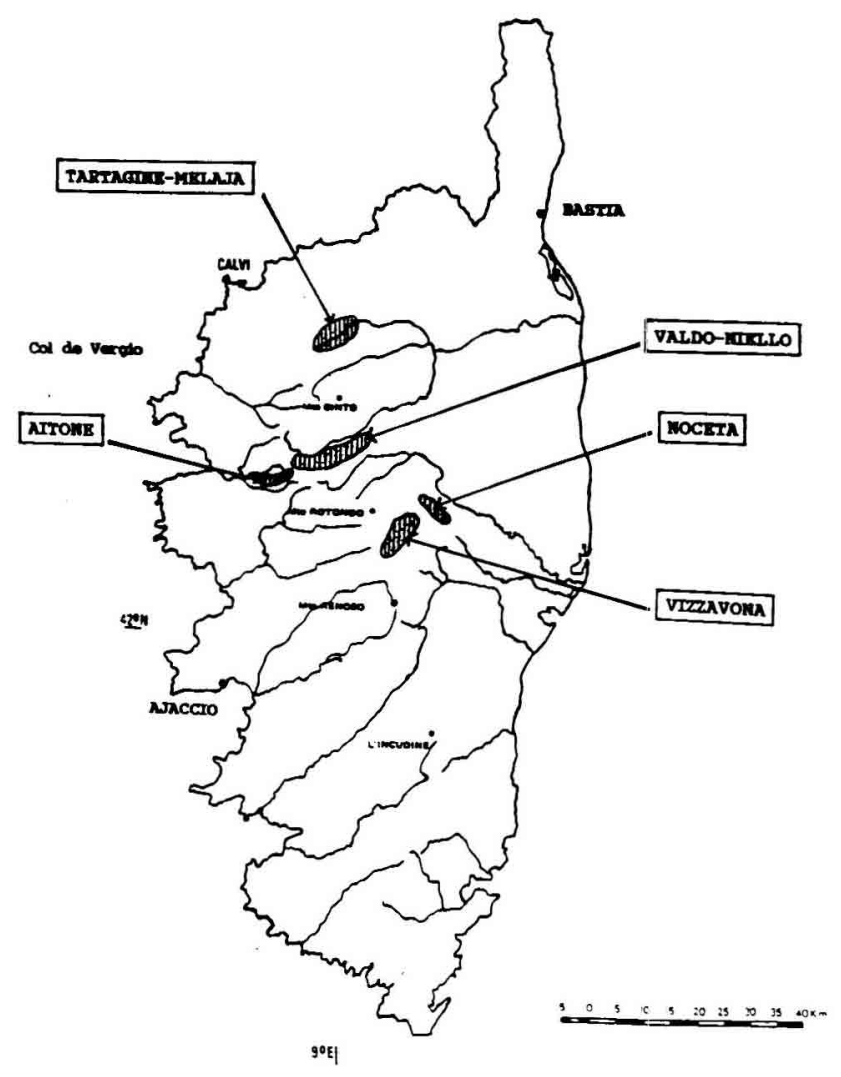

Fig. 1. Localisation des cinq massifs naturels corses étudiés.

- des mesures individuelles de hauteur totale annuelle $(\mathrm{HT})$, exprimées en $\mathrm{cm}$. Celles-ci ont permis le calcul d'accroissements périodiques en hauteur, respectivement entre 2 et 5 ans de plantation (IH 2-5), 5 et 11 ans de plantation (IH 5-11), et 2 et 11 ans de plantation (IH 2-11);

- une mesure individuelle de circonférence à $1,30 \mathrm{~m}$ (CIRC), exprimée en cm. L'aptitude à la croissance en diamètre s'exprimant plus tardivement (Arbez et Millier, 1972), celle-ci n'a été effectuée qu'après la onzième saison de végétation.

La qualité de branchaison de chaque individu en test a été appréciée, fin 1986, à l'aide d'une note globale évaluant le nombre et le type de défauts de fourchaison observés à la base des trois verticilles formés en 1983, 1984 et 1985 . L'arbre sans défaut recevait une note egale à 100; chaque ramicorne (") ou fourche (") observée retirait respectivement 5 et 10 points à cette valeur de base.

\section{Méthodes statistiques}

Après ajustement préalable des données aux facteurs contrôlés du milieu, les valeurs phénotypiques individuelles ont tout d'abord été

(") Les ramicornes et fourches sont définies comme des branches à angle d'insertion inférieur à $30^{\circ}$ dont le diamètre à la base est inférieur (ramicorne) ou voisin (fourche) de celui de la tige principale. 
décomposées selon un modèle d'analyse de variance hiérarchique mixte à deux facteurs, peuplement d'origine (fixe) et famille (aléatoire).

$$
Y_{i k}=\mu+p_{i}+F_{j(i)}+\varepsilon_{i k}
$$

où $\mu$ représente la moyenne générale du caractère $Y_{i}$

$p_{i}$ ef $F_{j(i)}$ sont respectivement les effets moyens des facteurs peuplement et famille dans peuplement;

\section{$\varepsilon_{i j k}$ est la résiduelle.}

Les moyennes des cinq peuplements naturels étudiés ont été comparées 2 à 2 à l'aide de la méthode de Bonferroni.

Les comparaisons de structure de la variabilité phénotypique intrapeuplement reposent sur l'utilisation d'un modèle d'analyse de variance à un facteur famille, considéré comme aléatoire.

$$
Y_{i j k}=\mu_{i}+F_{j(i)}+\varepsilon_{i j k}^{\prime}
$$

où $\mu_{i}$ représente la moyenne du peuplement $i$; $F_{i(i)}$ est l'effet moyen de la famille $j$ échantillonnee dans le peuplement $i$;

$\varepsilon_{i j k}$ est la résiduelle.

Les cinq matrices de variances-covariances phénotypiques ont été décomposées selon la méthode d'Henderson III, en matrice de variances-covariances entre familles $\Sigma_{F i}$ et en matrices de variances-covariances résiduelles $\Sigma_{W i}$. Ces deux ensembles de matrices de dispersion ont été comparées entre elles à l'aide du test de Kullback (Legendre P. et Legendre L., 1979).

Pour $p$ matrices de variances-covariances $\Sigma_{i}$ entre $k$ caractères, avec $n_{i}$ répétitions présentes pour le peuplement $i$, l'hypothèse $H_{0}$ suivante est testée :

$$
\begin{aligned}
\Sigma_{1}=\Sigma_{2}=\ldots=\Sigma_{i}=\Sigma & \text { où } \Sigma=\sum_{i=1,}^{\mathrm{p}}\left(n_{i} \Sigma_{i}\right) / n \\
& \text { avec } n=\sum_{i=1}^{\mathrm{p}} n_{i} ;
\end{aligned}
$$

En posant,

$$
\Gamma=\prod_{i=1}^{p}\left(\operatorname{det}(\Sigma)^{n / 2} / \operatorname{det}\left(\Sigma_{i}\right)^{n / 2}\right)
$$

Sous l'hypothèse $H_{0}$, la statistique $(-2$ loge( $\Gamma)$ est distribuée asymptotiquement comme un $\chi^{2}$ à $k(k+1)(p-1) / 2$ degrés de liberté.

Estimation des paramètres génétiques

L'utilisation de covariances entre apparentés permet de relier la matrice de variances-covariances des effets additifs, $\Sigma_{A i}$, à celle des effets familles, $\Sigma_{F i}=1 / 4 \quad \Sigma_{A i}$, en supposant vérifiées les trois hypothèses suivantes :

- 1) les descendances maternelles obtenues par pollinisation libre en forêt sont composées exclusivement de demi-frères.

-2) la consanguinité moyenne des cinq peuplements étudiés est nulle.

- 3) les arbres-mères échantillonnés dans un même peuplement ne sont pas apparentés.

Ont donc pu être calculés, pour chaque peuplement :

- l'héritabilité individuelle $\mathrm{h}^{2}$ de chaque caractère $k$, définie comme le rapport de la variance génétique additive à la variance phénotypique totale $\sigma^{2} p$ (Falconer, 1974) :

$$
h^{2}=4 \sigma_{F}^{2}\left(\sigma_{F}^{2}+\sigma_{W}^{2}\right)
$$

Les intervalles de confiance des héritabilités ont été calculées à l'aide des formules développées par Becker (1985), en situation déséquilibrée, pour un modèie à un seul facteur aléatoire.

- le coefficient de corrélation génétique additive $r_{A}\left(k, k^{\prime}\right)$ qui mesure la dépendance du contrôle génétique de type additif des deux caractères $k$ et $k^{\prime}$ :

$$
r_{A}\left(k, k^{\prime}\right)=\operatorname{Cov}_{F}\left(k, k^{\prime}\right) / \sqrt{\sigma_{F(k)}^{2} \sigma_{F(k)}^{2}}
$$

II nous est apparu intéressant d'estimer tout d'abord les paramètres génétiques intrapeuplement, puis d'évaluer le niveau de signification des différences observées entre les 5 populations en ayant recours au test de Kullback calculé sur les variances-covariances interfamille. 


\section{Résultats}

Performances comparées des cinq massifs naturels corses

Le test $F$ de l'effet peuplement, calculé suivant le modèle hiérarchique (1) est significatif pour l'ensemble des caractères de vigueur et de forme étudiés (Tableau II).

La survie globale observée à 2 ans de plantation atteint à peine $82 \%$. Les jeunes semis âgés de deux ans à la plantation ont eu à souffrir des sécheresses estivales successives des années 1975 et 1976, et d'une forte concurrence herbacée. La dernière hauteur totale mesurée après 11 saisons de végétation n'atteignit en moyenne que $420 \mathrm{~cm}$. En effet, les conditions de fertilité rencontrées sur le site et les années climatiques peu favorables qui suivirent la plantation, ne permirent pas aux jeunes pins de retrouver un rythme de croissance élevé.

Les différences entre peuplements se marquent essentiellement sur la croissance en hauteur :
- les trois massifs de Tartagine, Vizzavona et Noceta présentent à la fois, les hauteurs totales à 2 ans les plus élevées, les accroissements en hauteur les plus forts et les circonférences à $1,30 \mathrm{~m}$ les plus importantes;

- le peuplement de Valdo-Niello se distingue de ce groupe de tête par une hauteur initiale plus faible et un rythme de croissance en hauteur plus lent. Sa performance moyenne en circonférence rejoint cependant celle des trois premiers peuplements;

- la dernière place des classements reste occupée par le massif d'Aïtone, au taux de survie particulièrement bas et au démarrage de croissance très lent. Ce peuplement, situé à plus de $1000 \mathrm{~m}$ d'altitude sur un versant très arrosé, semble supporter difficilement les sécheresses estivales successives des premières années de plantation du test.

Les défauts de fourchaison relevés sur l'ensemble du dispositif pour les trois mêmes années sont peu importants et les différences observées entre peuplements

Tableau II. Comparaison de la survie moyenne, des performances de croissance et des notes moyennes de fourchaison des cinq massifs naturels.

\begin{tabular}{llllllll}
\hline Caractère & $\begin{array}{l}\text { Survie.2 } \\
\%\end{array}$ & $\begin{array}{l}H T .2 \\
\mathrm{~cm}\end{array}$ & $\begin{array}{l}\text { IH2-5 } \\
\mathrm{cm}\end{array}$ & $\begin{array}{l}\text { IH 5-11 } \\
\mathrm{cm}\end{array}$ & $\begin{array}{l}I H 2-11 \\
\mathrm{~cm}\end{array}$ & $\begin{array}{l}\text { CIRC.11 } \\
\mathrm{cm}\end{array}$ & $\begin{array}{l}\text { FOUR } \\
83-85\end{array}$ \\
\hline $\begin{array}{l}\text { Test F (a) } \\
\begin{array}{l}\text { Moyenne } \\
\text { générale }\end{array}\end{array}$ & $3,2^{*}$ & $23,0^{* *}$ & $19,1^{* *}$ & $25,0^{* *}$ & $26,2^{* *}$ & $6,1^{* *}$ & $3,1^{*}$ \\
& 81,6 & 26,2 & 81,6 & 312,8 & 394,4 & 23,8 & 96,6 \\
Tart & $84,8^{\alpha}$ & $27,4^{\alpha}$ & $84,3^{\alpha}$ & $319,6^{\alpha}$ & $403,9^{\alpha}$ & $24,0^{\alpha}$ & $96,7^{\alpha \beta}$ \\
Vald & $81,2^{\beta}$ & $25,8^{\beta}$ & $80,5^{\beta}$ & $306,2^{\beta}$ & $386,7^{\beta}$ & $23,7^{\alpha}$ & $96,2^{\beta}$ \\
Aito & $78,1^{\beta}$ & $24,4^{\alpha}$ & $76,9^{\alpha}$ & $305,4^{\beta}$ & $382,3^{\beta}$ & $22,8^{\beta}$ & $97,1^{\alpha}$ \\
Vizz & $81,4^{\beta}$ & $26,9^{\alpha}$ & $83,8^{\alpha}$ & $317,5^{\alpha}$ & $401,3^{\alpha}$ & $24,0^{\alpha}$ & $97,2^{\alpha}$ \\
Noce & $83,0^{\alpha}$ & $26,7^{\alpha}$ & $83,8^{\alpha}$ & $321,5^{\alpha}$ & $403,9^{\alpha}$ & $24,1^{\alpha}$ & $96,1^{\beta}$ \\
\hline
\end{tabular}

(a) $F(4,92)$ pour SURVIE.2 et $F(4,4952)$ pour les caractères de croissance.

- Test significatif à $5 \%$

** Test significatif à $1 \%$

Les moyennes présentant une lettre $\alpha, \beta$ ou $\Gamma$ identique ne sont pas significativement différentes au seuil de $5 \%$ (Test de Bonferroni). 


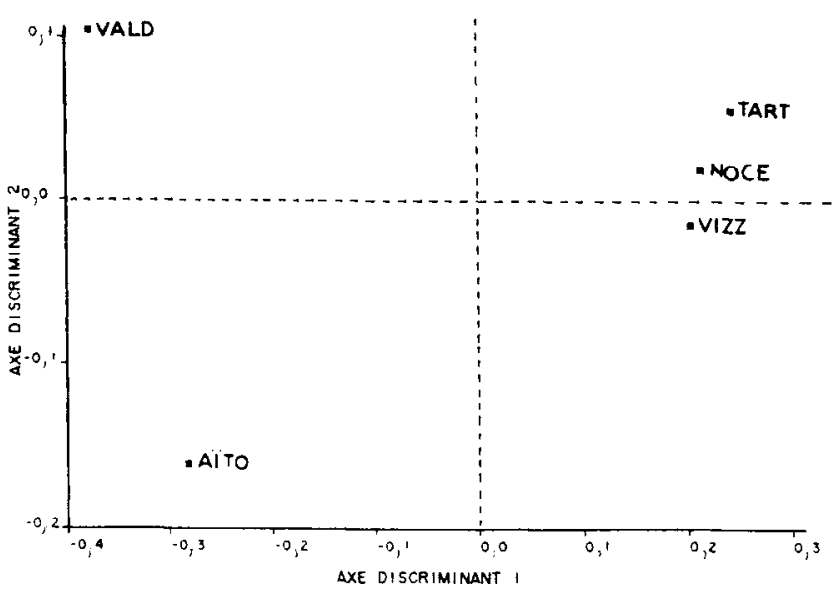

Fig. 2. Analyse factorielle discriminante du facteur peuplement. Représentation graphique des cinq massifs natureis selon les deux premiers axes de l'analyse.

ne sont significatives qu'au niveau $5 \%$ (Tableau II).

Seuls les arbres appartenant aux massifs d'Aïtone et de Vizzavona apparaissent moins défectueux que ceux originaires des massifs de Valdo-Niello et de Noceta.

La Fig. 2 présente les cinq peuplements corses dans le plan constitué par les deux premiers axes de l'analyse factorielle discriminante utilisant les 5 caractères suivants HT.2, IH 2-5, IH 5-11, FOUR 83-85 et CIRC.11. Les deux variables canoniques correspondantes tendent à définir trois groupes de populations.

L'inertie totale du nuage de points est essentiellement absorbée par le premier axe $(60,5 \%$ contre $20,1 \%$ pour l'axe 2$)$. Celui-ci, fortement corrélé aux caractères de croissance en hauteur (Tableau III), oppose les deux peuplements de ValdoNiello et d'Aïtone, aux trois autres massifs. Le deuxième axe utilise quant à lui la corrélation négative entre note de fourchaison et circonférence à $1,30 \mathrm{~m}$ pour séparer ces deux massifs géographiquement proches.

La confrontation de ces premiers résultats aux informations recueillies sur l'ensemble des tests de provenance, où sont représentés plusieurs peuplements naturels de lîle de Beauté, semble confirmer les deux points suivants (Roman-Amat, 1984) :

Tableau III. Corrélations canoniques entre les variables discriminantes et les variables initiales.

\begin{tabular}{llllll}
\hline $\begin{array}{l}\text { Variables } \\
\text { initiales }\end{array}$ & HT.2 & IH2-5 & IH5-11 & CIRC.11 & $\begin{array}{l}\text { FOUR } \\
83-85\end{array}$ \\
\hline $\begin{array}{l}\text { Axe 1 } \\
\text { V. canon. 1 }\end{array}$ & 0,816 & 0,837 & 0,977 & 0,375 & 0,204 \\
$\begin{array}{l}\text { Axe 2 } \\
\text { V. canon. 2 }\end{array}$ & 0,574 & 0,537 & 0,150 & 0,906 & $-0,740$ \\
\hline
\end{tabular}


Tableau IV. Tests $F$ et coefficients de variation interfamiliaux $\left(C V_{F}\right)$ des cinq massifs naturels corses pour quelques prédicteurs de la croissance en volume et de la forme.

\begin{tabular}{|c|c|c|c|c|c|c|}
\hline & & Tart & Vald & Aito & Vizz & Noce \\
\hline $\begin{array}{l}\text { Nb. familles } \\
\text { ddl intra }\end{array}$ & & $\begin{array}{r}20 \\
1042\end{array}$ & $\begin{array}{r}30 \\
1537\end{array}$ & $\begin{array}{r}20 \\
910\end{array}$ & $\begin{array}{r}12 \\
609\end{array}$ & $\begin{array}{r}15 \\
783\end{array}$ \\
\hline HT.2 & $\begin{array}{l}\text { (a) test } F \\
\mathrm{CV}_{F}\end{array}$ & $\begin{array}{l}4,96^{\star \star} \\
7,7 \%\end{array}$ & $\begin{array}{l}7,02^{* *} \\
9,7 \%\end{array}$ & $\begin{array}{c}7,70^{\star *} \\
11,9 \%\end{array}$ & $\begin{array}{l}4,05^{* *} \\
7,1 \%\end{array}$ & $\begin{array}{l}7,60^{* *} \\
9,9 \%\end{array}$ \\
\hline $\mathrm{IH} 2-5$ & $\begin{array}{l}\text { test } F \\
\mathrm{CV}_{\mathrm{F}}\end{array}$ & $\begin{array}{l}2,90^{* \star} \\
4,9 \%\end{array}$ & $\begin{array}{l}8,04^{* *} \\
9,6 \%\end{array}$ & $\begin{array}{l}5,18^{* *} \\
8,7 \%\end{array}$ & $\begin{array}{l}3,19^{\star *} \\
5,9 \%\end{array}$ & $\begin{array}{l}3,27^{* *} \\
5,7 \%\end{array}$ \\
\hline IH 5-11 & $\begin{array}{l}\text { test } F \\
\mathrm{CV}_{\mathrm{F}}\end{array}$ & $\begin{array}{l}6,10^{\star \star} \\
4,5 \%\end{array}$ & $\begin{array}{l}6,26^{* *} \\
5,2 \%\end{array}$ & $\begin{array}{l}7,04^{* *} \\
6,1 \%\end{array}$ & $\begin{array}{l}9,15^{* *} \\
6,1 \%\end{array}$ & $\begin{array}{l}6,69^{* *} \\
5,0 \%\end{array}$ \\
\hline IH 2-11 & $\begin{array}{l}\text { test } F \\
\mathrm{CV}_{\mathrm{F}}\end{array}$ & $\begin{array}{l}5,05^{\star *} \\
4,1 \%\end{array}$ & $\begin{array}{l}7,45^{* *} \\
5,9 \%\end{array}$ & $\begin{array}{l}6,95^{\star *} \\
6,5 \%\end{array}$ & $\begin{array}{l}7,81^{* *} \\
6,1 \%\end{array}$ & $\begin{array}{l}5,77^{*} \\
4,9 \%\end{array}$ \\
\hline CIRC.11 & $\begin{array}{l}\text { test } F \\
\mathrm{CV}_{\mathrm{F}}\end{array}$ & $\begin{array}{l}4,38^{* *} \\
6,2 \%\end{array}$ & $\begin{array}{l}6,67^{\star *} \\
8,7 \%\end{array}$ & $\begin{array}{c}6,99^{\star *} \\
10,3 \%\end{array}$ & $\begin{array}{l}4,23^{* *} \\
6,4 \%\end{array}$ & $\begin{array}{l}4,58^{\star *} \\
6,4 \%\end{array}$ \\
\hline $\begin{array}{l}\text { FOUR } \\
83-85\end{array}$ & $\begin{array}{l}\text { test } F \\
\mathrm{CV}_{\mathrm{F}}\end{array}$ & $\begin{array}{l}2,48^{* *} \\
1,4 \%\end{array}$ & $\begin{array}{l}1,69^{*} \\
1,1 \%\end{array}$ & $\begin{array}{l}1,67^{*} \\
0,9 \%\end{array}$ & $\underset{* * * * *}{1,18 \mathrm{~ns}}$ & $\begin{array}{l}2,31^{\star *} \\
1,5 \%\end{array}$ \\
\hline
\end{tabular}

(a) Test $F$ de l'effet famille pour chaque peuplement; ** significatif à $1 \%$; " significatif a $5 \%$; ns non significatif.

- aucune influence de la latitude n'est décelable sur les caractères de vigueur et de forme étudiés dans ce dispositif de la forêt d'Orléans;

- les deux massifs de Tartagine et de Noceta représentant les tranches altitudinales les plus faibles, semblent être plus vigoureux que les massifs de Valdo-Niello et d'Aïtone, situés à des altitudes plus élevées.

Etude de la variabilité génétique présente dans les cinq massifs corses

Les cinq massifs naturels corses ont pu être comparés sur les valeurs des paramètres génétiques des différents prédicteurs de la croissance en volume et de la forme, estimés grâce au modèle d'analyse de variance intrapeuplement (2).
Etude des coefficients de variation interfamille : $C V_{F}$

Les valeurs du test $F$ de l'effet famille et des coefficients de variation interfamille $C V_{F}$ ont été regroupées dans le Tableau IV pour les six caractères de l'étude et chacun des cinq massifs naturels corses.

La comparaison des matrices de variances-covariances résiduelles intrapeuplement $\Sigma_{W}$, réalisée à l'aide du test de Kullback, ne permet pas de rejeter l'hypothèse d'homogénéité des structures de variabilité intrafamille de ces cinq populations $\left(\chi^{2}(144 \mathrm{ddl})=70,2 \mathrm{~ns}\right)$. Les différents descripteurs de la variabilité interfamilles, estimés pour chaque peuplement, peuvent donc être comparés sans correction préalable.

L'effet famille testé sur l'ensemble des caractères de croissance est hautement 
significatif pour les cinq massifs naturels corses. Les coefficients de variation interfamille $C V_{F}$ sont cependant très variables d'une population à lautre, en particulier pour les descripteurs les plus juvéniles (HT.2, HT.5 et IH 2-5).

Pour tous ces caractères, il semble exister une liaison négative entre performance moyenne et niveau de variabilité interfamille.

La note moyenne de fourchaison observée entre 1983 et 1985 est peu variable d'une descendance à l'autre, quel que soit le massif étudié. L'effet famille sur ce caractère est même non significatif pour le peuplement de Vizzavona représenté, il est vrai, par un petit nombre de descendances.
Etude des paramètres génétiques : $h^{2}$ et $r_{A}$

Les estimations des héritabilités individuelles des six caractères étudiés sont présentées dans le Tableau $V$ avec leur intervalle de confiance calculé au seuil de $5 \%$. Leur ont été ajoutées les estimations des corrélations génétiques additives entre deux prédicteurs de la croissance en volume, et la note moyenne de fourchaison.

Si les héritabilités individuelles calculées pour chacun des cinq peuplements semblent varier du simple au double pour un même caractère, il n'en reste pas moins que le degré de précision de ces estimations reste plutôt faible dès que le nombre de familles échantillonnées est inférieur à 30 .

Tableau V. Paramètres génétiques intrapeuplement estimés pour quelques prédicteurs de la croissance en volume et de la forme.

\begin{tabular}{llllll}
\hline & $\begin{array}{l}\text { Tart } \\
\text { 20 fam. }\end{array}$ & $\begin{array}{l}\text { Vald } \\
30 \mathrm{fam} .\end{array}$ & $\begin{array}{l}\text { Aito } \\
20 \mathrm{fam} .\end{array}$ & $\begin{array}{l}\text { Vizz } \\
12 \mathrm{fam} .\end{array}$ & $\begin{array}{l}\text { Noce } \\
15 \mathrm{fam} .\end{array}$ \\
\hline $\mathrm{h}^{2}$ (HT.2) & 0,28 & 0,42 & 0,51 & 0,22 & 0,44 \\
& {$[0,21-0,68]$} & {$[0,27-0,67]$} & {$[0,31-0,90]$} & {$[0,10-0,58]$} & {$[0,25-0,89]$} \\
$h^{2}$ (IH 2-5) & 0,14 & 0,47 & 0,33 & 0,16 & 0,16 \\
& {$[0,08-0,37]$} & {$[0,32-0,74]$} & {$[0,18-0,57]$} & {$[0,05-0,48]$} & {$[0,08-0,46]$} \\
$h^{2}$ (IH 5-11) & 0,34 & 0,37 & 0,46 & 0,54 & 0,38 \\
& {$[0,20-0,62]$} & {$[0,25-0,56]$} & {$[0,28-0,83]$} & {$[0,28-1,00]$} & {$[0,22-0,76]$} \\
$h^{2}$ (IH 2-11) & 0,27 & 0,44 & 0,46 & 0,47 & 0,33 \\
& {$[0,14-0,52]$} & {$[0,29-0,71]$} & {$[0,28-0,83]$} & {$[0,24-1,00]$} & {$[0,17-0,70]$} \\
$h^{2}$ (CIRC.11) & 0,24 & 0,40 & 0,46 & 0,23 & 0,25 \\
& {$[0,13-0,48]$} & {$[0,26-0,64]$} & {$[0,28-0,83]$} & {$[0,10-0,60]$} & {$[0,13-0,57]$} \\
$h^{2}$ (FOUR 83-85) & 0,11 & 0,05 & 0,05 & $* * *$ & 0,10 \\
& {$[0,04-0,26]$} & {$[0,01-0,13]$} & {$[0,01-0,18]$} & $* * *$ & {$[0,03-0,28]$} \\
$r_{A}$ (CIRC-FOUR 11) & $-0,29$ & $-0,21$ & $-0,31$ & $* * *$ & $-0,88$ \\
$r_{A}$ (IH-FOUR 2-11) & $-0,30$ & 0,08 & $-0,16$ & $* * *$ & $-0,55$ \\
\hline
\end{tabular}

* L'intervalle de confiance de l'héritabilité individuelle estimée est donné au seuil de 5\%. 
Les quatre remarques générales suivantes peuvent néanmoins être formulées :

- tous les prédicteurs de la croissance en volume étudiés dans les cinq peuplements peuvent être considérés comme héritables $\left(h^{2}>0.2\right)$. Les différences observées entre massifs semblent plus importantes pour les caractères juvéniles (HT.5, IH 25);

- la note moyenne de fourchaison présente une héritabilité souvent proche de 0 ce qui dénote un déterminisme génétique de type additif peu important voire inexistant pour ce caractère, quel que soit le peuplement étudié. Ces résultats rejoignent des observations antérieures réalisées sur quelques peuplements artificiels de Sologne de pin laricio de Corse (Portefaix, 1987);

- pour tous les peuplements où une variabilité interfamiliale de la note de fourchaison a pu être mise en évidence, la corrélation génétique additive entre ce caractère et la circonférence à $1,30 \mathrm{~m}$ est négative. Chez de nombreuses essences forestières, la croissance en diamètre semblerait être fortement corrélée à la vigueur de la cime (Arbez, comm. pers.). En augmentant la biomasse des parties aériennes, le développement de défauts de fourchaison favoriserait cette composante de la croissance en volume.

Compte tenu du faible nombre de familles utilisé pour ces estimations intrapeuplements, cette corrélation n'est, toutefois, fortement défavorable, que pour le massif de Noceta.

- la liaison génétique reliant prédicteurs de la croissance en hauteur et note de fourchaison semble être moins étroite, mais reste toujours fortement défavorable, pour le peuplement de Noceta. Si elle reste aux alentours de -0.20 pour les deux massifs de Tartagine et d'Aitone, elle devient nulle pour le peuplement de Valdo-Niello.

Test de l'hornogénéité des matrices de variances-covariances interfamilles

Le niveau de signification des différences observées entre paramètres génétiques des cinq peuplements est précisé par le test d'homogénéité des matrices de

Tableau VI. Tests d'homogénéité des variances interfamilles de quelques prédicteurs de la croissance en volume et de la forme.

\begin{tabular}{lllc}
\hline $\begin{array}{l}\text { Valeur du test } \\
\text { de Kullback }\end{array}$ & $\begin{array}{l}\text { Test } 1 \alpha \\
N_{f} \text { réel }\end{array}$ & $\begin{array}{l}\text { Test } 2^{\beta} \\
30 \text { familles }\end{array}$ & $\begin{array}{l}N_{f} r \\
\text { minimum }\end{array}$ \\
\hline HT.2 (a) & $1,59 \mathrm{~ns}$ & $3,20 \mathrm{~ns}$ & 87 \\
IH 2-5 (a) & $5,27 \mathrm{~ns}$ & $7,98 \mathrm{~ns}$ & 36 \\
IH 5-11(a) & $1,17 \mathrm{~ns}$ & $1,91 \mathrm{~ns}$ & $>100$ \\
IH 2-11 (a) & $1,91 \mathrm{~ns}$ & $3,10 \mathrm{~ns}$ & 90 \\
CIRC.11 (a) & $2,93 \mathrm{~ns}$ & $5,13 \mathrm{~ns}$ & 55 \\
FOUR 83-85 (b) & $5,13 \mathrm{~ns}$ & $4,05 \mathrm{~ns}$ & 58 \\
\hline
\end{tabular}

(a) Comparaison des cinq peuplements : $\chi^{2}(4,5 \%)=9,49 \chi^{2}(4,1 \%)=13,3$.

(b) Comparaison des quatre peuplements : $\chi^{2}(3,5 \%)=7,81 \chi^{2}(3,1 \%)=11,3$.

$\alpha$ Valeur du test pour le nombre exact de familles échantillonnées par peuplement.

$\beta$ Valeur du test pour 30 familles échantillonnées par peuplement.

$\Gamma$ Nombre moyen de familles à échantillonner pour rejeter l'hypothèse d'homogénéité des cinq variances interfamilles estimées. 
Tableau VII. Discrimination des cinq massifs corses sur la base des matrices de variances-covariances interfamilles.

\begin{tabular}{|c|c|c|c|c|c|c|}
\hline $\begin{array}{l}\text { Combinaison } \\
\text { de variables }\end{array}$ & $\begin{array}{l}\text { Test } \chi^{2} \\
\text { global }\end{array}$ & $\begin{array}{l}\text { Comp } \\
\text { deux }\end{array}$ & $\begin{array}{l}\text { son desp } \\
I x\left(\chi^{2}\right)\end{array}$ & lements & & \\
\hline $\begin{array}{c}\text { Etape I } \\
\text { IH 2-5 } \\
\text { IH 5-11 }\end{array}$ & 23,70 (a) & $\begin{array}{l}\text { Aito } \\
\text { Vald } \\
\text { Vizz } \\
\text { Noce }\end{array}$ & $\begin{array}{c}\text { Tart } \\
4,43 \\
7,70 \\
12,64^{* *} \\
0,84\end{array}$ & $\begin{array}{l}\text { Aito } \\
2,68 \\
9,28^{*} \\
1,33\end{array}$ & $\begin{array}{l}\text { Vald } \\
\begin{array}{c}15,10^{* *} \\
4,35\end{array}\end{array}$ & Vizz \\
\hline $\begin{array}{l}\text { Etape II } \\
\text { HT.2 } \\
\text { IH 5-11 } \\
\text { FOUR 83-85 }\end{array}$ & 30,90 (b) & $\begin{array}{l}\text { Aito } \\
\text { Vald } \\
\text { Noce }\end{array}$ & $\begin{array}{c}\text { Tart } \\
3,95 \\
22,09^{\star *} \\
6,85\end{array}$ & $\begin{array}{l}\text { Aito } \\
\begin{array}{c}13,48^{*} \\
5,93\end{array}\end{array}$ & $\begin{array}{l}\text { Vald } \\
15,84^{*}\end{array}$ & \\
\hline $\begin{array}{l}\text { Etape III } \\
\text { HT.2 } \\
\text { IH 5-11 } \\
\text { IH 2-11 } \\
\text { CIRC.11 } \\
\text { FOUR 83-85 }\end{array}$ & $\underset{* \star}{99,72(c)}$ & $\begin{array}{l}\text { Aito } \\
\text { Noce }\end{array}$ & $\begin{array}{l}\text { Tart } \\
28,80^{* *} \\
11,26\end{array}$ & $\begin{array}{l}\text { Aito } \\
23,12^{* *}\end{array}$ & & \\
\hline
\end{tabular}

(a) Comparaison des cinq peuplements : $\chi^{2}(12,5 \%)=21,0 \chi^{2}(12,1 \%)=26,2$.

(b) Comparaison de quatre peuplements : $\chi^{2}(18,5 \%)=28,9 \chi^{2}(18,1 \%)=34,8$.

(c) Comparaison de trois peuplements : $\chi^{2}(30,5 \%)=43,8 \chi^{2}(30,1 \%)=50,9$.

variances-covariances interfamilles, calculé à l'aide de la fonction de Kullback.

Les résultats présentés dans le Tableau VI correspondent aux comparaisons des cinq variances de chacune des variables. Ils soulignent qu'aucun caractère ne permet à lui seul de différencier les cinq populations naturelles. Les écarts observés entre estimations ne seraient significatifs que si le nombre de familles représentant chaque peuplement était beaucoup plus élevé.

La prise en compte de combinaisons particulières de variables a néanmoins révélé des différences de variabilité interfamille entre peuplements. La démarche analytique présentée ici se limite aux comparaisons les plus discriminantes. A chaque étape, celles-ci correspondent aux combinaisons de variables conduisant aux valeurs de $\chi^{2}$ les plus élevées pour un nombre minimal de caractères pris en compte (Tableau VII). La Fig. 3 schématise les trois tests de dissemblance significatifs obtenus, sans toutefois indiquer de véritables distances entre les 5 peuplements.

L'analyse du dendrogramme obtenu et les estimations de variances-covariances interfamilles utilisées permettent de caractériser les structures génétiques des cinq peuplements naturels de la façon suivante :

\section{Vizzavona :}

- aucune variabilité interfamille de la note moyenne de fourchaison n'a pu être mise en évidence pour ce massif du centre de l'île de Beauté;

- la variance interfamille de la croissance en hauteur reste faible pendant toute la crise de plantation, pour s'accroitre considérablement lors de la deuxième phase de croissance. 


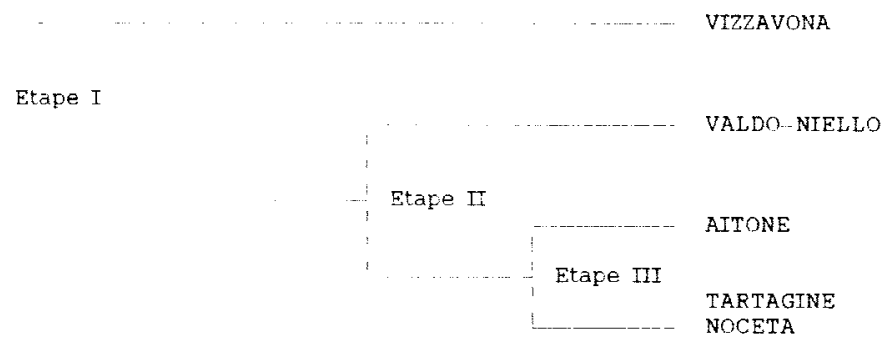

Fig. 3. Dendrogramme obtenu à partir des tests d'homogénéité des matrices de variances-covariances interfamilles.

Valdo-Niello : c'est le seul peuplement naturel qui présente, pour la note moyenne de fourchaison, à la fois une certaine variabilité interfamilles et une liaison génétique additive non défavorable avec les prédicteurs de la croissance en hauteur.

Aïtone : ce massif situé à plus de $1000 \mathrm{~m}$ d'altitude se caractérise par une forte variabilité interfamilles de tous les caractères de vigueur : croissance en hauteur pour les deux phases successives étudiées et circonférence à $1,30 \mathrm{~m}$

Tartagine et Noceta : ces deux peuplements n'ont pu être distingués l'un de l'autre sur la base de la structure de la variabilité interfamilles des six caractères étudiés. Leurs corrélations génétiques additives entre note moyenne de fourchaison et circonférence à $1,30 \mathrm{~m}$ seraient significativement différentes si les deux populations étaient représentées par plus de 30 familles.

La différenciation obtenue à partir de caractères morphologiques est de même ampleur que celle observée par BonnetMasimbert et Bikay-Bikay (1978) sur ces mêmes cinq peuplements grâce à l'analyse du polymorphisme allélique de 4 loci de la glutamate-oxaloacétate-transaminase.

La prise en compte des origines géographiques et des performances moyennes en test de ces cinq peuplements conduit à la formulation des deux remarques essentielles suivantes:

- 1) il n'y a pas de recoupement entre proximité géographique et homogénéité des structures de variabilité génétique, estimées sur des caractères morphologiques. Les deux peuplements d'Aitone et de Valdo-Niello, séparés par le seul col de Vergio sont considérés ici comme deux populations naturelles bien distinctes. A l'opposé, les deux massifs de Tartagine et de Noceta, aux structures génétiques voisines, sont situés dans deux vallées séparées par plus de 70 kilomètres de distance;

-2) il semble exister une liaison entre les performances moyennes en test et le niveau de variabilité interfamilles estimé. Ainsi, Aïtone occupe la dernière place des classements établis sur les différents caractères de vigueur, tout en présentant les variances génétiques additives les plus élevées pour ces mêmes prédicteurs. A linverse, Tartagine et Noceta sont les plus performants sur toute la période de plantation et ont les plus faibles estimations de variances interfamilles.

Seule, la recherche des facteurs pouvant affecter la structure génétique d'une population naturelle permettrait d'expliquer ces deux observations. 


\section{Discussion et conclusion}

Parmi les théories explicatives de l'évolution de la structure génétique des espèces forestières anémophiles, celle défendue par S. Wright (1977) s'adresse aux essences pour lesquelles une importante différenciation génétique entre populations voisines a pu être mise en évidence. Dans ces unités, installées le plus souvent dans des sites écologiques isolés, plusieurs facteurs concourent à une limitation des flux géniques:

- la taille effective réduite de la population conduit à une dérive génétique au fil des générations;

- l'existence de relations privilégiées entre arbres reproducteurs proches et une dispersion des graines sur de faibles distances (environ $50 \mathrm{~m}$ ) expliquent la création de cercles de voisinage présentant un taux moyen de consanguinité élevé (Müller, 1974);

- les échanges géniques entre populations voisines sont limités par l'existence de barrières écologiques ou phénologiques.

Seules les pressions de sélection naturelle parviennent à limiter l'augmentation du taux moyen de consanguinité et accentuent la spécialisation adaptative des différentes populations géographiques. Chez les espèces forestières essentiellement allogames, la dépression de consanguinité est, en effet, particulièrement élevée pour tous les caractères adaptatifs : taux de graines pleines, taux de germination, survie des jeunes semis et croissance au stade juvénile (Burrows, 1982; Jouve, 1983; Yazdani et al., 1985).

Plusieurs études, engagées sur quelques espèces de la famille des Pinaceae, originaires de régions épargnées par les glaciations successives du Quaternaire ont montré que la différenciation entre populations voisines peut être importante (Gullberg et al., 1985).

En supposant que le pin laricio de Corse corresponde à une de ces espèces particulières, il reste à préciser quels pourraient être les rôles respectifs des pressions de sélection naturelle et de la dérive génétique dans la différenciation des cinq peuplements naturels de cette étude.

\section{Rôle des pressions de sélection naturelle}

Si les sols sur lesquels sont installés les cinq massifs forestiers sont peu variés, il existe une grande diversité des régimes pluviométriques rencontrés suivant l'exposition et l'altitude. Un tel constraste pourrait, en partie, expliquer les différences de croissance et de survie observées en forêt d'Orléans, entre les peuplements de la tranche altitudinale 750-1 $000 \mathrm{~m}$ et de versant sec (Tartagine, Noceta), et les massifs installés à plus de $1000 \mathrm{~m}$ sur versants très arrosés (Valdo-Niello, Aïtone).

\section{Rôle de la dérive génétique}

Les différences observées entre les cinq estimations de variances génétiques additives pourraient trouver leur origine dans des phénomènes de dérive génétique conduisant à une augmentation du taux moyen de consanguinité des populations naturelles isolées. Parmi les facteurs susceptibles de conduire, au contraire, à une réduction rapide et notable de ce taux peuvent être relevés :

- les incendies : ils affectent le plus souvent la partie basse des peuplements, où le pin laricio de Corse voisine avec le pin maritime. Les arbres épargnés par le feu seraient principalement pollinisés par l'ensemble des reproducteurs du peuplement voisin, la dispersion du pollen et des graines se trouvant facilitée par la création 
de grandes trouées. Au cours de la régénération du peuplement, les recombinaisons génétiques se trouveraient ainsi favorisées et conduiraient à une diminution du niveau moyen de consanguinité. Les estimations de variances génétiques additives seraient alors peu élevées. Un tel scénario a pu être démontré pour quelques populations de Pinus ponderosa (Woods et al., 1983);

- la colonisation de nouvelles surfaces en limite inférieure des peuplements : la rupture des cercles de voisinage, existant dans la population adjacente, conduirait également à une diminution des variances génétiques additives;

- l'action humaine : l'influence d'une telle pression évolutive ne peut être écartée, mais ne pourrait être que limitée, compte tenu de la longueur de l'intervalle de génération du pin laricio dans son aire naturelle (> 100 ans) et de la gestion sylvicole de ces forêts de montagne, encore difficilement accessibles.

II faut noter que les facteurs limitant les effets de la dérive génétique concernent essentiellement les peuplements de basse altitude et que, faute de données historiques suffisantes, il est impossible de déterminer la cause exacte de la diminution des variances génétiques additives. Ces hypothèses peuvent néanmoins être utilisées pour expliquer les différences de structure génétique observées entre les cinq peuplements corses étudiés :

- Aïtone, correspondant à la tranche altitudinale d'échantillonnage la plus élevée, présente bien les plus fortes estimations de variances génétiques additives. De plus, les faibles taux de survie et les rythmes de croissance juvénile relativement lents observés en test de descendances pourraient, en partie, s'expliquer par l'expression d'une forte dépression de consanguinité;
- à l'opposé, les structures génétiques additives peu variables de Tartagine et de Noceta traduiraient une rupture partielle des cercles de voisinage établis jusqu'alors dans ces deux massifs installés entre 750 et $900 \mathrm{~m}$ d'altitude. De nombreux incendies ont en effet touché ces peuplements depuis le début du siècle et probablement, depuis plusieurs générations d'arbres;

- les situations observées pour les peuplements' de Valdo-Niello et Vizzavona sont plus difficiles à interpréter, peut-être, car cette étude intervient à une phase intermédiaire du cursus évolutif de ces deux populations.

Cette contribution à la description de la variabilité génétique présente dans l'aire naturelle du pin laricio de Corse devra être enrichie par de nouvelles investigations. Parmi elles, peuvent être citées :

- l'exploration des forêts plus méridionales situées à l'est du Monte Renoso (Ghisoni, Marmano) et dans le massif de l'Incudine (Bavella, Marghese). Cette étude préciserait l'ampleur de la différenciation des populations naturelles à l'échelle de l'île de Corse. Certaines origines se sont, de plus, révélées être particulièrement performantes en test de provenances (RomanAmat, 1984) et mériteraient une étude plus approfondie de leur valeur génétique;

- l'évaluation du taux moyen de consanguinité des peuplements permettrait d'évaluer le rôle du mode de reproduction en forêt naturelle sur l'évolution des structures génétiques. Par cet intermédiaire, de nouvelles connaissances seraient apportées sur la nature de l'effet altitudinal mis en évidence sur les caractères de croissance. Les effets d'adaptation aux conditions environnementales pourraient être différenciés de ceux liés à une variation clinale de la consanguinité suivant ce même gradient. Toutes ces informations permettront ainsi de préciser les normes 
d'échantillonnage intrapeuplement à adopter en forêt naturelle de pin laricio de Corse.

\section{Références}

Arbez M. \& Millier C. (1972) Variabilité, héritabilité et corrélations entre caractères chez de jeunes pins laricio de Calabre (Pinus nigra Arn. ssp. laricio var. calabrica). Conséquence et problèmes des indices de sélection. Proceedings of the IUFRO Genetics SABRAO Joint Symposium. Tokyo

Baradat P. (1976) Genetics of monoterpenic and sesquiterpenic hydrocarbons in maritime pine, Pinus pinaster Ait. IUFRO Joint Meeting on Advanced Generation Breeding Bordeaux, $6 \mathrm{pp}$.

Becker W.A. (1985) Manual of quantitative genetics. Academic Enterprises Puliman, Washington

Bergmann F. (1974) Genetischer Abstand zwischen europaischer Fichtenpopulationen (Picea abies) auf der Basis von IsoenzymGennäufigkeiten. Silvae Genet. 23, 28-32

Birot Y. \& Christophe C. (1979) Genetic variation within and between populations of Douglas fir. Silvae Genet. 28 (5-6), 197-206

Birot Y. \& Christophe C. (1983a) Genetic structures and expected genetic gains from multitrait selection in wild populations of Douglas fir and Sitka spruce. I - Genetic variation between and within population. Silvae Genet. 32 (5-6), 141150

Birot Y. \& Christophe C. (1983b) Genetic structures and expected genetic gains from multitrait selection in wild population of Douglas fir and Sitka spruce. II - Practical application of index selection on several populations. Silvae Genet. 32 (5-6), 173-180

Bonnet-Masimbert M. \& Bikay-Bikay V. (1978) Variabilité intraspécifique des isozymes de la glutamate-oxaloacétate-transaminase chez Pinus nigra Arnold. Intérêt pour la taxonomie des sous-espèces. Silvae Genet. 27 (2), 71-79

Burrows P.M. (1982) Experimental inbreeding depression. Forest Sci. 28 (1), 148-156

Cotterill P.P. \& James J.W. (1984) Number of offsprings and plot sizes required for progeny testing. Silvae Genet. 33 (6), 203-209
El Kassaby Y.A. Yeh F.C. \& Sziklai O. (1981)

Estimation of the outcrossing rate of Douglas fir allozyme polymorphisms. Silvae Genet. 30 (6), 182-184

Falconer D.S. (1974) Introduction à la génétique quantitative. Masson, Paris

Gullberg U., Yazdani R., Rudin D. \& Ryman N. (1985) Allozyme variation in Scots Pine (Pinus sylvestris L.) in Sweden. Silvae Genet. 34 (6), 193-201

Guyon J.P. (1980) Variabilité géographique et écophysiologique du pin maritime. Mémoire de fin d'études a l'Ecole Nationale des ingénieurs des Travaux des Eaux et Forêts, Nogent-surVernisson

Jouve P. (1983) Etude de la dépression de consanguinité chez le pin maritime (Pinus pinaster Ait.). Mémoire de DAA

Legendre L. \& Legendre P. (1984) Ecologie numérique. Tome 2 : La structure des données écologiques. Masson, Paris

Müller G. (1974) Some population genetic aspects derived from new results of pollen and seed dispersal of Spruce and Pine respectively. Proceedings of the IUFRO Joint Meeting Population and Ecological Genetics, breeding theory and progeny testing, Stockholm

Portefaix C. (1987) Défaut de fourchaison chez le pin laricio de Corse (Pinus nigra Arn. ssp. laricio var. crosicana Loud) : décomposition du phénomène en vue de l'amélioration génétique. Ann. Sci. For. 44 (4), 435-454

Rehfeldt G.E. (1982) Differentiation of Larix occidentalis populations from the Northern Rocky Mountains. Silvae Genet. 31 (1), 13-19

Roman-Amat B. (1984) Contribution à l'exploration et à la valorisation de la variabilité intraspécifique et individuelle du pin laricio de Corse, Pinus nigra Arn. ssp. laricio var. corsicana Loud. Thèse de Docteur Ingénieur. Université de Paris Sud. Centre d'Orsay $n^{\circ} 679$

Rudin D. (1976) Biochemical genetics and selection application of isoenzymes in tree breeding. In proceedings of the IUFRO Joint Meeting on Advanced Generation Breeding, Bordeaux, 145-164

Squillace A.E. (1976) Biochemical genetics and selection components of volatile terpenes. In proceedings of the IUFRO Joint Meeting on Advanced Generation Breeding, Bordeaux, 167-178 
Tigerstedt P.M.A. (1982) Composition of neighbouring effect in a naturally regenerating population of Scots pine. Silvae Fennica 16, 122-129

Woods J.H., Blake G.M. \& Allendorf F.W. (1983) Amount and distribution of isozyme variation in ponderosa Pine from eastern Montana. Silvae Genet. 32 (5-6), 151-156

Wright S. (1977) Evolution and the genetics of populations. Vol. 3, Experimental results and evolutionnary deductions. University of Chicago Press, Chicago

Yang J.C., Ching T.M. \& Ching K.K. (1977) Isozyme variation of coastal Douglas fir. I - A study of geographic variation in the enzyme systems. Silvae Genet. 26 (1), 10-18

Yazdani R., Muona O., Rudin D. \& Szmidt A.E. (1985) Genetic structure of Pinus sylvestris L. seed-tree stand and naturally regenerated understory. Forest Sci. 31 (2), 430-436 